\title{
Case Report Use of Direct Carotid Artery Puncture Access for Flow Diverter Embolization Combined with Coil Embolization: A Case Report
}

Yusuke Funakoshi, Taketo Hatano, Mitsushige Ando, Hideo Chihara, Wataru Takita, Keisuke Tokunaga, Takuro Hashikawa, Takahiko Kamata, Eiji Higashi, and Izumi Nagata

Objective: The flow diverter (FD) was developed as a new approach for treating aneurysms. However, FD embolization requires high technical skills and is challenging when the access to the aneurysm is difficult and when sufficient force cannot be transmitted to the catheter because of severe vascular tortuosity. For FD embolization, when a transfemoral approach is too difficult, we perform direct puncture of the common carotid artery (CCA) under direct visualization by making a small incision. Herein, we report our experience using this approach.

Case Presentation: We present a case of an 80-year-old woman with an unruptured aneurysm on the right internal carotid artery (ICA) treated by FD embolization in conjunction with coil embolization. We considered that access to the aneurysm would be challenging, with limited catheter maneuverability because of severe tortuosity of the bilateral femoral arteries and the region from the right CCA to the ICA. Thus, we decided to access the aneurysm by direct puncture of the right CCA. The aneurysm was easily accessed using this approach, and a stable procedure was completed by placing a sheath with a detachable hemostasis valve and then switching the hemostasis valve to a Y-connector on the sheath. As good catheter control was obtained despite the vascular tortuosity, the Pipeline Flex could be deployed and placed at the appropriate position.

Conclusion: Direct puncture of the CCA under direct visualization for FD embolization is useful when access to the aneurysm is challenging and when catheter maneuverability decreases because of severe tortuosity.

Keywords > direct puncture, common carotid artery, flow diverter embolization, coil embolization, unruptured aneurysm

\section{Introduction}

Since its pharmaceutical approval in April 2015, the Pipeline Flex flow diverter (FD) embolization device has become indicated for unruptured or chronic ruptured cerebral aneurysms with a neck diameter $\geq 4 \mathrm{~mm}$ and a maximum

Department of Neurosurgery, Stroke Center, Kokura Memorial Hospital, Kitakyushu, Fukuoka, Japan

Received: September 3, 2018; Accepted: October 2, 2018 Corresponding author: Yusuke Funakoshi. Department of Neurosurgery, Stroke Center, Kokura Memorial Hospital, 3-2-1 Asano, Kokurakita, Kitakyushu, Fukuoka 802-8555, Japan

Email: sf1wan0610@gmail.com

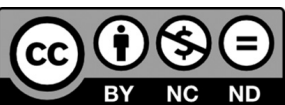

This work is licensed under a Creative Commons Attribution-NonCommercialNoDerivatives International License.

(C)2019 The Japanese Society for Neuroendovascular Therapy aneurysm diameter $\geq 10 \mathrm{~mm}$ located from the petrous portion of the internal carotid artery (ICA) to the superior hypophyseal artery. The long-term outcomes for safety and efficacy of this device were recently reported. ${ }^{1)}$ Recent studies have also described the treatment outcomes for the FD in ruptured aneurysms, aneurysms with a maximum diameter $<10 \mathrm{~mm}$, and aneurysms of the posterior circulation, ${ }^{2-4)}$ and an expansion of indications is anticipated in the near future.

The FD is technically difficult to use, ${ }^{5)}$ and even greater skills may be required when sufficient force cannot be transmitted to the catheter because of severe vascular tortuosity. For FD embolization procedures performed at our institution, when a transfemoral approach is deemed difficult, we perform direct puncture of the common carotid artery (CCA) under direct visualization by making a small incision. Herein, we report a case of an unruptured ICA aneurysm in which direct puncture of the CCA was useful 


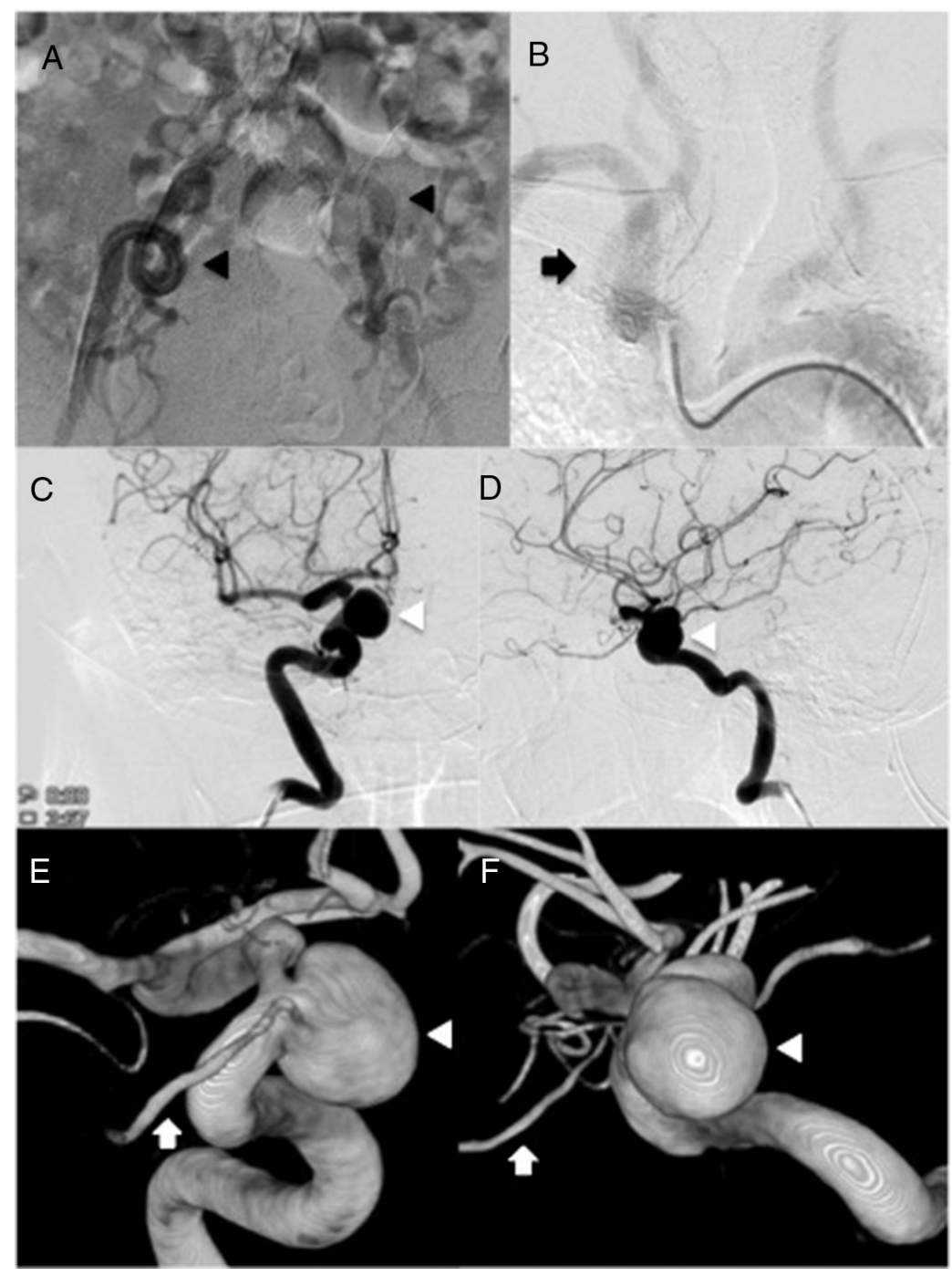

Fig. 1 Preoperative digital subtraction angiography. (A and B) The bilateral femoral artery (black arrowhead) and the right CCA (black arrow) are severely tortuous because of arteriosclerotic changes. (C-F) The right ICA is severely tortuous and there is a saccular aneurysm (white arrowhead; maximum dome diameter of $13.4 \mathrm{~mm}$ and neck diameter of $5.2 \mathrm{~mm}$ ) protruding on the medial side of the bifurcation of the right ICA and the ophthalmic artery (white arrow). CCA: common carotid artery; ICA: internal carotid artery

for FD embolization performed in conjunction with coil embolization.

\section{Case Presentation}

An 80-year-old woman was found to have an unruptured aneurysm on the right ICA during MRI performed as part of a detailed examination for dementia by a previous physician. The patient did not exhibit any cognitive function issues, but wished to undergo aneurysm treatment, and was thus referred to our department. On digital subtraction angiography, the bilateral femoral artery, and the CCA were severely tortuous because of arteriosclerotic changes (Fig. 1A and 1B). The right ICA was also severely tortuous (Fig. 1C and 1D). A saccular aneurysm with a maximum dome diameter of $13.4 \mathrm{~mm}$ and neck diameter of $5.2 \mathrm{~mm}$ was detected, which protruded on the medial side of the bifurcation of the right ICA and the ophthalmic artery (Fig. 1C-1F). The diameter of the distal ICA was $4.8 \mathrm{~mm}$, and the proximal was $5.1 \mathrm{~mm}$. There was moderate stenosis (2.3 $\mathrm{mm}$ diameter) at the distal side of the ICA with the aneurysm. Based on the size and location of the aneurysm, FD embolization was indicated, and coil embolization was also used to avoid delayed cerebral aneurysm rupture. 


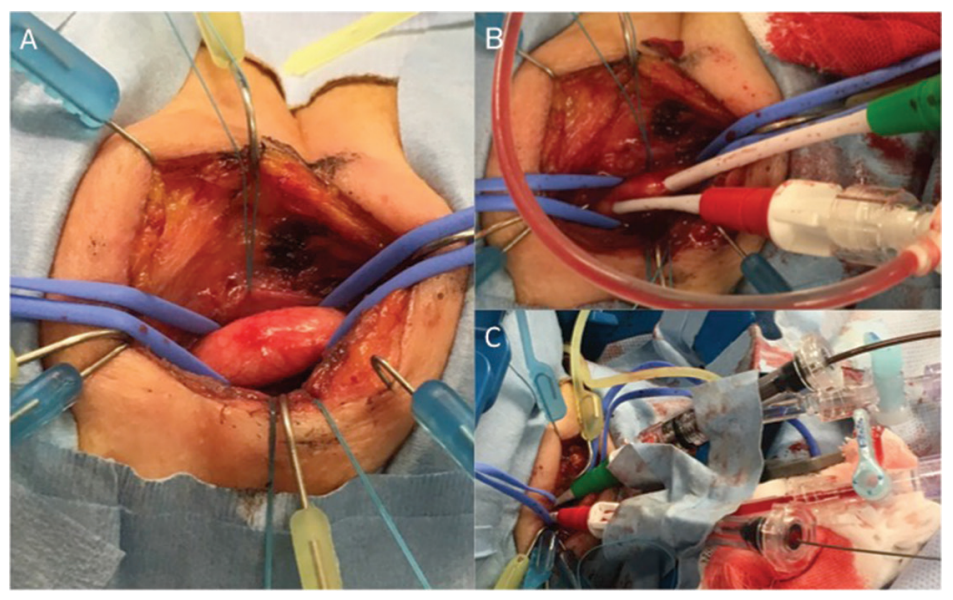

Fig. 2 Intraoperative photographs. (A) The right CCA is exposed and secured with vascular tape. (B) A 6-Fr sheath and a 4-Fr sheath with detachable hemostasis valves are inserted into the right CCA. (C) Hemostasis valves on the sheaths are replaced with a Y-connector, and the procedure is continued while maintaining heparinized saline perfusion. CCA: common carotid artery

Aspirin (100 mg/day) and clopidogrel (75 mg/day) were started 2 weeks before surgery. We recognized that accessing the aneurysm may be challenging and that catheter maneuverability may be limited because of the severe tortuosity of the bilateral femoral arteries and the region from the right CCA to the ICA. Thus, we decided to access the aneurysm by direct puncture of the right CCA.

\section{Endovascular treatment}

After inducing general anesthesia, a transverse incision (approximately $4 \mathrm{~cm}$ ) was made along the skin crease in the right neck. The CCA was exposed while carefully performing hemostasis with a bipolar device (Fig. 2A). The CCA was then punctured with a needle under direct visualization, a guidewire was advanced to the CCA, and a 6-Fr sheath with a detachable hemostasis valve (Medikit Peripheral Marker Sheath; Medikit, Tokyo, Japan) was placed for FD embolization. Using the same technique, a 4-Fr sheath with a detachable hemostasis valve for coil embolization was also placed (Fig. 2B). Hemostasis valves on the sheaths were replaced with a Y-connector, and the procedure was continued while maintaining heparinized saline perfusion (Fig. 2C). From the 6-Fr sheath, a 5-Fr distal support catheter (Navien intracranial support catheter; Medtronic Neurovascular, Irvine, CA, USA) and a microcatheter for introducing the FD (Marksman micro catheter; Medtronic Neurovascular) were advanced distally beyond the aneurysm (Fig. 3A). From the 4-Fr sheath, a microcatheter for coil insertion (Echelon10, ev3; Covidien, Irvine, CA,
USA) was placed in the aneurysm, and the Pipeline Flex $(5.0 \mathrm{~mm} \times 25 \mathrm{~mm}$; Medtronic Neurovascular) was deployed (Fig. 3B). However, the microcatheter for coil insertion interfered with the Pipeline Flex, and its tip turned toward the aneurysmal wall. Consequently, the Pipeline Flex was re-sheathed and the tip of the microcatheter for coil insertion was re-shaped in a circular shape along the circumference within the aneurysm. While making small adjustments to the position of each catheter, the Pipeline Flex was deployed and placed across the neck (Fig. 3C and $\mathbf{3 G}$ ). However, a portion of the Pipeline Flex did not fully deploy because of the microcatheter used for coil insertion. Intra-aneurysmal embolization was performed with five coils, consisting of one Axium 3D $10.0 \mathrm{~mm} \times 30 \mathrm{~cm}(\mathrm{ev} 3$; Covidien), one Axium 3D $9.0 \mathrm{~mm} \times 30 \mathrm{~cm}$, and three Axium 3D $8.0 \mathrm{~mm} \times 20 \mathrm{~cm}$ coils (Fig. 3D and 3E). Intraaneurysmal stagnation of contrast medium was confirmed (Fig. 3F). Finally, percutaneous transluminal angioplasty was performed with a Transform SC $4 \times 7 \mathrm{~mm}$ (Stryker, Kalamazoo, MI, USA) at the distal and proximal side of the ICA with the aneurysm, where Pipeline Flex deployment was insufficient (Fig. 3G-3I). The sheath was removed, the puncture site was sutured, and the wound was closed.

\section{Postoperative course}

Postoperatively, no obvious neurologic deficits appeared, and formation of a hematoma at the puncture site was not detected. Although postoperative MRI showed a small signal hyperintensity on the outside of the head of the 


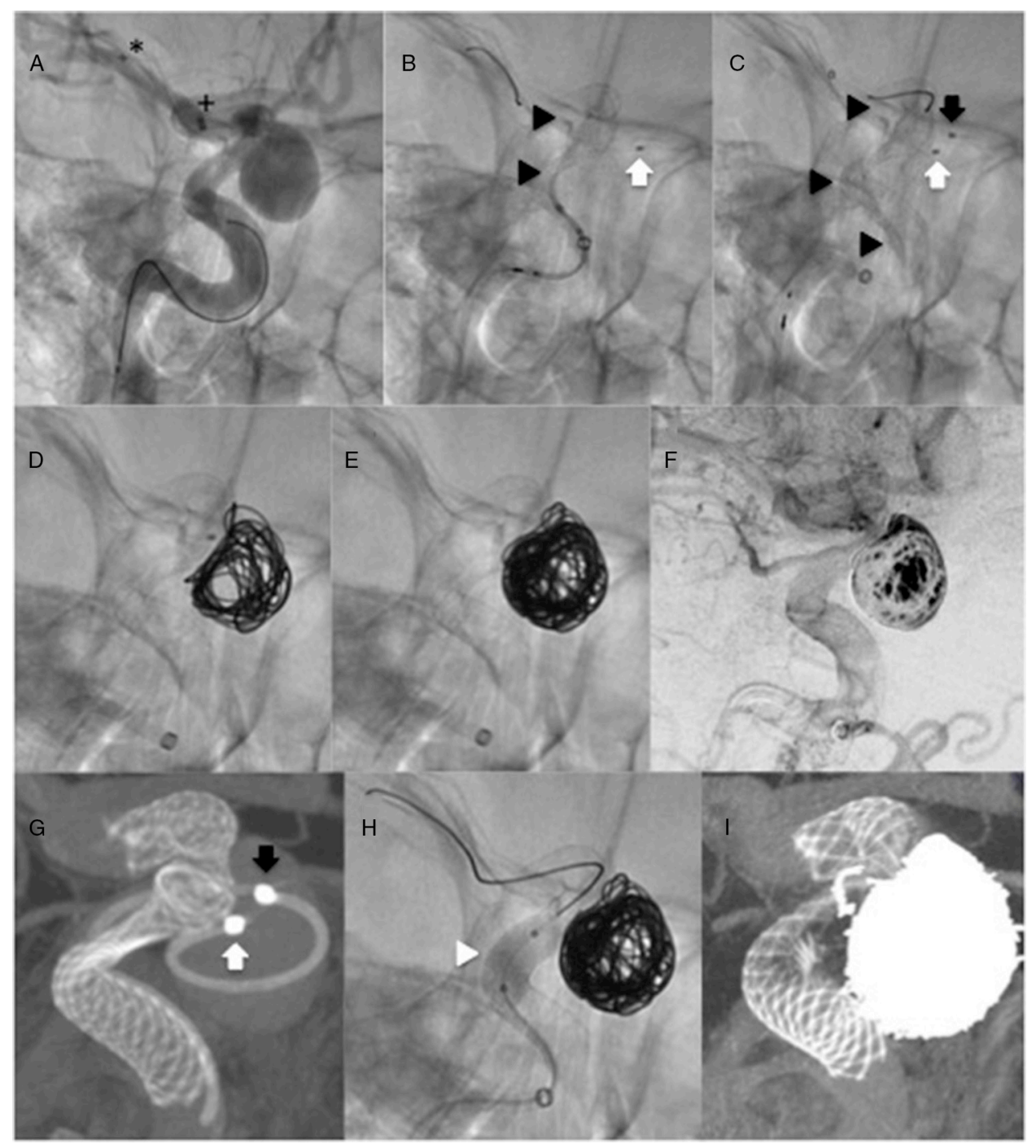

Fig. 3 Intraoperative digital subtraction angiography. (A) A 5-Fr distal support catheter (*) and a microcatheter for introducing the FD (+) are advanced distally beyond the aneurysm. (B) A microcatheter for coil insertion is placed in the aneurysm (first-marker: white arrow), and the Pipeline Flex (black arrowheads) is deployed. (C) The tip of the microcatheter for coil insertion is shaped in a circular shape along the circumference within the aneurysm (first-marker, white arrow; second marker, black arrow) and the Pipeline Flex (black arrowheads) is deployed and placed across the neck. (D-F) Intra-aneurysmal embolization is performed with five coils, with confirmation of intra-aneurysmal stagnation of contrast medium. (G) 3D angiography image shows that a portion of the Pipeline Flex is not fully deployed because of the microcatheter for coil insertion. (H) Percutaneous transluminal angioplasty is performed with balloon catheter (white arrowhead) where Pipeline Flex deployment is insufficient. (I) 3D angiography image showing that the Pipeline Flex deployment is sufficient. FD: flow diverter

right caudate nucleus on diffusion- weighted imaging (Fig. 4A), no obvious aneurysms were visualized on MRA (Fig. 4B and 4C). The patient did not develop any complications, and she was subsequently discharged.

\section{Discussion}

The FD embolization device is being increased used clinically in some Japanese institutions. However, handling of the device is technically difficult, necessitating use by surgeons with a high degree of experience in intra cerebrovascular treatments, as well as appropriate training prior to use. ${ }^{5)}$ Further technical skills may be necessary when sufficient force cannot be transmitted to the catheter because of severe vascular tortuosity. For this reason, when using FD embolization at our institution, we perform the procedure with direct puncture of the CCA under direct visualization by making a small incision to the neck when 1) the vascular 


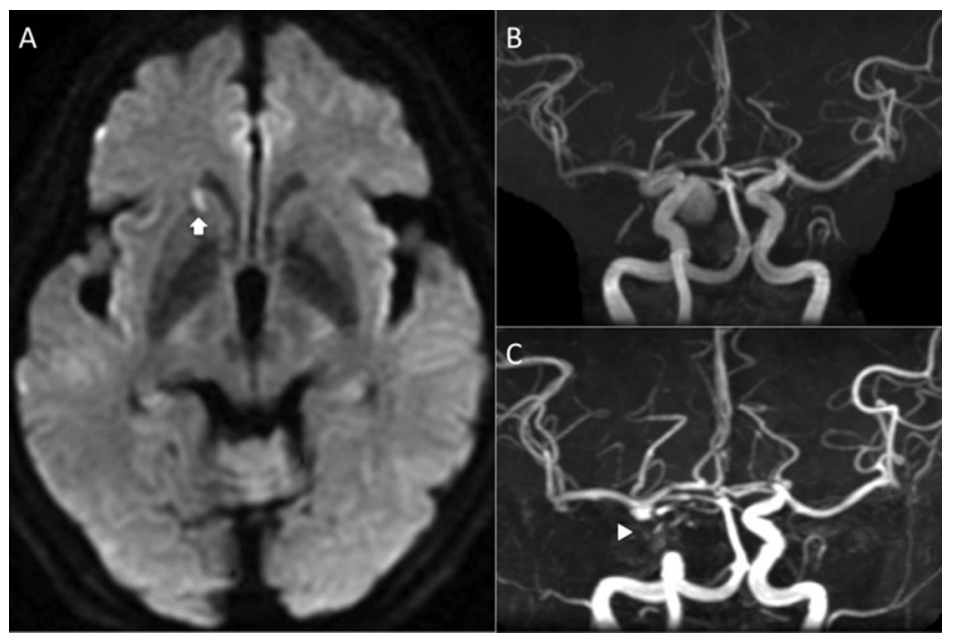

Fig. 4 Postoperative MRI. (A) There is a small signal hyperintensity on the outside of the head of the right caudate nucleus on diffusion-weighted imaging (white arrow). (B) Preoperative MRA shows a saccular aneurysm on the medial side of the right ICA. (C) Despite interference by the Pipeline Flex, no obvious aneurysms are observed (white arrowhead). ICA: internal carotid artery

tortuosity is so severe that a transfemoral approach is inherently infeasible or 2) decreased catheter maneuverability and the occurrence of forced maneuvers is considered likely. In fact, we select direct puncture of the CCA when a catheter cannot be induced to the ICA in preoperative DSA. Direct puncture of the CCA is effective in cases where a transfemoral approach is difficult, and is performed in various endovascular treatments including acute revascularization, 6,7) carotid artery stenting, ,,9) and cerebral aneurysm coil embolization. ${ }^{10,11)}$ Angiospasm and vascular dissection have been reported as complications of direct puncture of the $\mathrm{CCA},{ }^{7,10)}$ although hematoma formation often becomes the primary issue in endovascular treatments that require antiplatelet drug administration or systemic heparinization. ${ }^{6-11)}$ When a hematoma forms around the CCA, dyspnea may arise from compression of the airway. Oshima et al. ${ }^{8)}$ recommended direct puncture of the CCA under direct visualization by making a small incision rather than the percutaneous approach, to make certain of the puncture and hemostasis, and that a sheath is passed through the subcutaneous tissue to be fixed. At our institution, we have used this technique in three cases of FD embolization, including the present case, and have not encountered issues with hematoma formation. Although a skin incision of approximately $4 \mathrm{~cm}$ was necessary, accurate puncture of the CCA can be performed under direct visualization. We fix a sheath with tape, rather than through subcutaneous tissue, as the puncture angle is not limited. Improving hemostasis is also possible by suturing the puncture site after sheath removal. To prevent postoperative bleeding from surrounding soft tissues, ensuring hemostasis using a bipolar device from the skin incision step is important.

In the present case, we considered that the approach to the aneurysm may be challenging, and that catheter maneuverability would be decreased because of severe tortuosity of the bilateral femoral arteries and the region from the right CCA to the ICA. Thus, we accessed the aneurysm by direct puncture of the CCA via a cervical incision. The aneurysm was easily accessed using this approach, and a stable procedure could be completed by placing a sheath with a detachable hemostasis valve and switching the hemostasis valve to a Y-connector on the sheaths. Furthermore, as good catheter control was obtained without being affected by vascular tortuosity, the Pipeline Flex could be deployed and placed at the appropriate position.

The risk of serious complications such as delayed cerebral aneurysm rupture is high in FD embolization for large aneurysms, symptomatic aneurysms, aneurysms with a dome-to-neck ratio $>1.6$, and aneurysms that exhibit a jet flow pattern of inflowing contrast medium. ${ }^{5,12}$ In such cases, coil embolization is sometimes utilized in combination with FD. ${ }^{5,13)}$ In the present case, the dome-to-neck ratio was high at 2.58, and the aneurysm had protruded into the dura mater. Thus, we determined that the risk for subarachnoid hemorrhage because of delayed cerebral aneurysm rupture was high, and used concurrent coil embolization. The microcatheter for FD advancement and the microcatheter for coil insertion must be advanced from separate guiding 
catheters to avoid interference. When this was performed with direct puncture to the CCA under direct visualization, simultaneous placement of the two sheaths at the exposed CCA and advancement of the microcatheters from each sheath were achieved, allowing treatments while maintaining maneuverability.

\section{Conclusion}

Direct puncture of the CCA under direct visualization for FD embolization is useful when access to the aneurysm is challenging and when catheter maneuverability decreases because of severe tortuosity.

\section{Disclosure Statement}

The authors declare that they have no conflict of interest concerning the materials and methods used in this study and the findings specified in this paper.

\section{References}

1) Chiu $\mathrm{AH}$, Cheung $\mathrm{AK}$, Wenderoth JD, et al: Long-term follow-up results following elective treatment of unruptured intracranial aneurysms with the pipeline embolization device. AJNR Am J Neuroradiol 2015; 36: 1728-1734.

2) McAuliffe $W$, Wenderoth JD: Immediate and midterm results following treatment of recently ruptured intracranial aneurysms with the Pipeline embolization device. AJNR Am J Neuroradiol 2012; 33: 487-493.

3) Kallmes DF, Hanel R, Lopes D, et al: International retrospective study of the pipeline embolization device: a multicenter aneurysm treatment study. AJNR Am J Neuroradiol 2015; 36: $108-115$.
4) Srinivasan VM, Ghali MGZ, Reznik OE, et al: Flow diversion for the treatment of posterior inferior cerebellar artery aneurysms: a novel classification and strategies. J Neurointerv Surg 2018; 10: 663-668.

5) Oishi H, Teranishi K, Yatomi K, et al: [Paradigm shift of endovascular therapy after clinical introduction of a flow diverter for large intracranial aneurysms.] Jpn J Neurosurg (Tokyo) 2017; 26: 104-111. (in Japanese)

6) Miyamoto M, Ishi Y, Ajiki M, et al: [A case of the direct carotid artery puncture in endovascular thrombectomy.] JNET 2015; 9: 170-174. (in Japanese)

7) Mokin M, Snyder KV, Levy EI, et al: Direct carotid artery puncture access for endovascular treatment of acute ischemic stroke: technical aspects, advantages, and limitations. J Neurointerv Surg 2015; 7: 108-113.

8) Oshima R, Kondo R, Nagahata M, et al: [Carotid artery stenting by direct puncture of carotid artery through a small skin incision: case report.] JNET 2012; 6: 122-126. (in Japanese)

9) Guimaraens L, Theron J, Casasco A, et al: Carotid artery stenting by direct percutaneous puncture. J Vasc Surg 2011; 54: 249-251.

10) Blanc R, Piotin M, Mounayer C, et al: Direct cervical arterial access for intracranial endovascular treatment. Neuroradiology 2006; 48: 925-929.

11) Nii K, Kazekawa K, Onizuka M, et al: Direct carotid puncture for the endovascular treatment of anterior circulation aneurysms. AJNR Am J Neuroradiol 2006; 27: 1502-1504.

12) Kulcsár Z, Houdart E, Bonafé A, et al: Intra-aneurysmal thrombosis as a possible cause of delayed aneurysm rupture after flow-diversion treatment. AJNR Am J Neuroradiol 2011; 32: 20-25.

13) Rouchaud A, Brinjikji W, Lanzino G, et al: Delayed hemorrhagic complications after flow diversion for intracranial aneurysms: a literature overview. Neuroradiology 2016; 58: 171-177. 